\title{
Structure and Properties of Layered Ti-6Al-4V-Based Materials Fabricated Using Blended Elemental Powder Metallurgy
}

\author{
S.V.Prikhodko ${ }^{1}$, O.M.Ivasishin ${ }^{2}$, P.E.Markovsky², D.G.Savvakin ${ }^{2}$, O.O.Stasiuk ${ }^{2}$ \\ ${ }^{1}$ Department of Materials Science and Engineering, University of California Los Angeles, Los Angeles, CA 90095, \\ USA \\ ${ }^{2}$ G.V. Kurdyumov Institute for Metal Physics, National Academy of Science of Ukraine, 36, Vernadsky Blvd., 03142, \\ Kiev, Ukraine
}

\begin{abstract}
Due to the high specific strength of Ti, materials on its base are indispensable when high-strength and low-weight requests are a chief demand from the industry. Reinforcement of Ti-alloys with hard and light particles of TiC and $\mathrm{TiB}$ is a credible pathway to make metal matrix composites (MMC) with enhanced elastic moduli without compromising the material's low-weight. However, reinforcement of the alloy with hard particles inevitably lowers the value of toughness and plasticity of material. Yet, in many applications simultaneous high hardness and high plasticity are not required through the entire structure. For instance, parts that need enhanced wear resistance or resistance upon ballistic impact demand high hardness and strength at the surface, whereas their core necessitates rather high toughness and ductility. Such combination of mechanical properties can be achieved on layered structures joining two and more layers of different materials with different chemical composition and/or microstructure within each individual layer.
\end{abstract}

Multi-layered structures of Ti-6Al-4V alloy and its metal-matrix composites (MMC) with 5 and10\% (vol.) of TiC and TiB were fabricated in this study using blended elemental powder metallurgy (BEPM) of hydrogenated Ti. Postsintering hot deformation and annealing were sometimes also employed to improve the microstructure and properties. Structure of materials were characterized using light optical microscopy, scanning electron microscopy, electron backscattered diffraction, x-ray microscopy, tensile and 3-point flexural tests. The effect of various fabrication parameters was investigated to achieve desirable microstructure and properties of layered materials. Using optimized processing parameters, relatively large multilayered plates were made via BEPM and demonstrate superior antiballistic performance compared to the equally sized uniform Ti-6Al-4V plates fabricated by traditional ingot and wrought technology.

\section{Introduction}

The anti-ballistic protection of land systems, mobility and protection of the fighting vehicles and military personnel is vital in success of defense and anti-terrorist operations. Traditional material for armor is rolled homogeneous or high strength steel $[1,2]$. Use of steel armor, however, can increase the overall weight of the fighting vehicle on $15-20 \%$, which causes change in vehicle mobility, maneuverability, fuel efficiency and requires stronger breaks and more powerful engines [3]. The Army is in search of lightweight substitute for steel armor. Due to the high specific strength of titanium, materials on its base are contemplated as a viable alternative in low-weight armor production [3, 4]. However, when the armor parts are fabricated using traditional ingot and wrought technology the feasibility of 
implementation is questionable due to the high cost of the armor parts. In this regard the use of blended elemental powder metallurgy (BEPM) of Ti offers an effective cost reduction due to its ability to produce near-net shape parts, while the waste is considerably reduced [5]. Besides, BEPM can facilitate reinforcement of Ti-alloys with hard and light particles of $\mathrm{TiC}$ and $\mathrm{TiB}$ to make metal matrix composites (MMC) with enhanced elastic moduli without compromising the material's low-weight so desirable for armor. Reinforcement of the alloy with hard particles, however, most likely lowers the value of toughness and plasticity of material. Yet, in many applications, including armor, simultaneous high hardness and high plasticity are not essential through the entire structure: armor parts require high hardness and strength at the surface, whereas their core rather necessitates high toughness and ductility. Such combination of mechanical properties can be achieved on layered structures joining two and more layers of different materials with different chemical composition and/or microstructure within each individual layer. The objective of this study was development of cost-efficient technology for fabrication of low-weight and superior antiballistic properties multilayered structures (ML) made using BEPM of Ti-6Al-4V (wt.\%) alloy (Ti-64) and composites on its base with $\mathrm{TiB}$ and TiC.

\section{Materials and Experiments}

Combinations of high strength and enhanced ductility for anti-ballistic applications can be attained in the same part by creation of layered structures, which combine at least two materials with different properties. In manufacturing of armor, it is common to use a layered material consisting of a front-facing layer, whose purpose is to blunt and abrade the incoming projectile and a back-facing supporting layer absorbing energy by deformation. In our armor design we choose to combine layers of Ti-64, which provide sufficient ductility, along with metal matrix composites (MMC) on a Ti-64 base reinforced with $\mathrm{TiC}$ or $\mathrm{TiB}$ particles to provide high hardness. Layers with higher concentrations of reinforcement particles have greater hardness. These high hardness layers build the front of the armor plate, to enhance wear resistance and to deform and stop projectiles upon ballistic impact. The Ti-64 alloy layer with higher ductility and toughness forms the backside of the plate to prevent crack propagation and plate destruction. For ML structures with more than two layers, we performed different tests with having the hardest layer in the middle of the plate to prevent extensive spallation of this most brittle layer and in the front of the plate to maximize the abrasion of the projectile.

ML structures of Ti-64 alloy and its MMC with 5 and 10\% (vol.) of TiC and TiB were fabricated using BEPM of hydrogenated Ti. Four different powder sets of titanium hydride $\mathrm{TiH}_{2}(3.5 \% \mathrm{H}$, wt. $)$ with different particles sizes $(<40,<100,80-100,100-125 \mu \mathrm{m})$ was used to determine the effect of the base powder size on shrinkage behavior and sintering characteristics. $\mathrm{TiH}_{2}$ powder was blended with corresponding amounts of a master alloy powder, $60 \% \mathrm{Al}-$ $40 \% \mathrm{~V}$ (wt.), with particles size smaller than $63 \mu \mathrm{m}$ to form the required Ti-64 composition. To form the MMC layers the required amounts of reinforcement particles powders were added and mixed before the pressing stage. The size of $\mathrm{TiC}$ powder particles used was $1-30 \mu \mathrm{m}$. In order to obtain $\mathrm{TiB}$ inclusions as a part of the composite, we used $\mathrm{TiB}_{2}$ powder with 1-20 $\mu \mathrm{m}$ size particles expected to chemically transform during the sintering via the following reaction: $\mathrm{TiB}_{2}+\mathrm{Ti}=2 \mathrm{TiB}$. For the ML structures, the blends for each layer were prepared separately and added to the die before pressing. The die pressing (DP) and sintering technique were used to fabricate the samples. Plates, with the size $90 \times 90 \times 18 \mathrm{~mm}$ were pressed at $150 \mathrm{MPa}$; bars $65 \times 10 \times 10 \mathrm{~mm}$ and cylinders $10 \mathrm{~mm}$ diameters, $10-12 \mathrm{~mm}$ height were pressed at 150 and $640 \mathrm{MPa}$. Plates were suitable for ballistic test and bars and cylinders for mechanical tests and structure characterization. Single layer materials were fabricated to test the properties of individual layers. Sintering of all preform samples was conducted in a vacuum furnace $\left(1250^{\circ} \mathrm{C}, 4 \mathrm{~h}\right)$ followed by slow furnace cooling. Postsintering hot deformation and annealing were also tested. 
Structures of materials were characterized using light optical microscopy, scanning electron microscopy, electron backscattered diffraction and x-ray microscopy. Mechanical properties of materials have been evaluated using hardness, tensile and 3-point flexural tests. The deformation energy was measured based on engineering stress-strain curves obtained on tensile test. Ballistic test was performed in the NATO certified laboratory at Ivan Chernyakhovsky National University of Defense (Ukraine). The ballistic test conditions: B-32 bullet (caliber $7.62 \times 54 \mathrm{~mm}$, mass 10.4 g), kinetic energy 3500-3800 J. More details on samples fabrication and characterization protocols used in this study are discussed elsewhere $[6,7]$.

\section{Results and Discussion}

Light optical microscopy (Fig 1) and the SEM images obtained on smaller size samples pressed at $640 \mathrm{MPa}$ show the grain size of the alloys is generally below $100 \mu \mathrm{m}$. The grain size for the composite layers is commonly smaller (Fig 2). We see nice compaction and good integration between the layers. EBSD results of TiB composite show presence of three different phases: HCP $\alpha-\mathrm{Ti}, \mathrm{BCC} \beta-\mathrm{Ti}$, and orthorombic TiB shown in Fig 2 (c) in red, blue and yellow correspondingly.

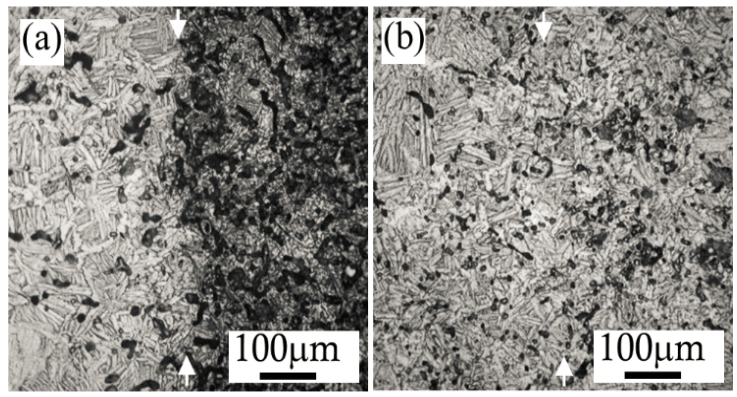

Fig. 1. Light optical micrographs of bi-layered structures fabricated using DP at $640 \mathrm{MPa}$ followed by sintering $\left(1250^{\circ} \mathrm{C}, 4 \mathrm{hrs}\right.$ ). Images show interface (arrowed) between the layers of Ti-64 alloy (left) and MMC on its base (right): with $10 \% \mathrm{TiB}$ in (a) and $5 \%$ of $\mathrm{TiC}$ in (b).

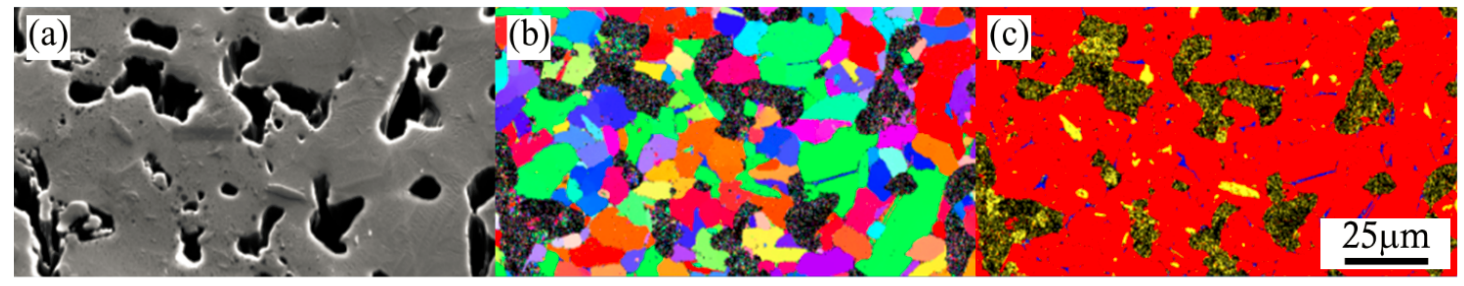

Fig. 2. SEM images of MMC with TiB particles. (a) Secondary electron image; EBSD orientation map (b) shows grains in a variety of crystallographic orientations. Phase map (c) shows three phases: hexagonal close-packed $\alpha-$ $\mathrm{Ti}$ (red), body-centered cubic $\beta$-Ti (blue) and orthorhombic TiB (yellow). All images are taken at the same magnification.

All layered samples of large dimension suitable for the ballistic test (plates) demonstrate significant linear shrinkage after the sintering. That was result of dehydrogenation of Ti and particles' sintering. Plates with TiC composite, 
however, were successfully made using die-pressing protocol. That was due to very close values of shrinkage of the alloy and TiC composites layers. Shrinkage values measured on plates were 15.7, 15.5 and 15.1\% for Ti-64, Ti$64+5 \% \mathrm{TiC}$ and Ti-64+10\%TiC layers correspondingly. Insignificant shrinkage mismatch of individual layers didn't cause cracking or distortion of the plates due to the minor interface stress which most likely was compensated by variation in porosity of adjacent layers.

Table 1. Residual porosity, Vickers hardness and tensile properties of individual layers.

\begin{tabular}{|c|c|c|c|c|c|}
\hline \# & Material & Porosity, \% & $\begin{array}{c}\text { Hardness, } \\
\text { HV }\end{array}$ & UTS, MPa & Elong., \% \\
\hline 1 & Ti-64 & $1.8 \%$ & 345 & 1034 & 7.2 \\
\hline 2 & Ti-64 + 5TiC & $3.8 \%$ & 333 & 708 & $<1$ \\
\hline 3 & Ti-64 + 10TiC & $3.6 \%$ & 373 & 618 & 0 \\
\hline 4 & Ti-64 & $3.6 \%$ & 299 & 969 & 8.3 \\
\hline 5 & Ti-64 + 5TiB & $4.5 \%$ & 327 & 847 & 0 \\
\hline 6 & Ti-64 + 10TiB & $8 \%$ & 324 & 512 & 0 \\
\hline 7 [9] & Ti-64 & $1.5-1 \%$ & $320-340$ & $940-1000$ & $10-14$ \\
\hline
\end{tabular}

Situation with the sintering of ML plates with TiB composite was quite different. Those plates demonstrate very different shrinkage values of individual layers: 13.5, 11 and $8.5 \%$ for Ti-64, Ti-64+5\%TiB and Ti-64+10\%TiB correspondingly. Measured shrinkage mismatch builds up the interfacial stress between the layers during the sintering and causes significant shape change of the plates and their cracking and delamination. In order to evaluate those negative effects, we performed detailed structure analysis and dilatometry study of individual powder compacts during continuous heating. 


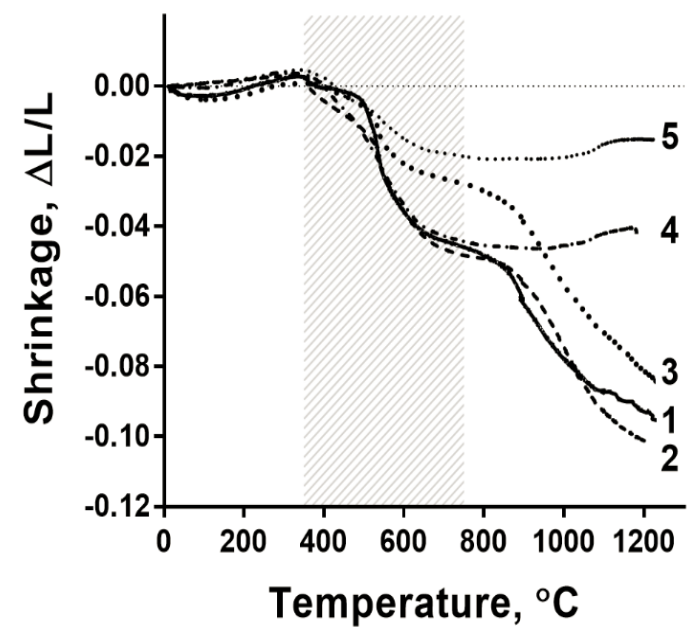

Fig. 3. Dilatometry heating curves for $\mathrm{TiH}_{2}$ powder compact (1) and compacted powder blends on the base of $\mathrm{TiH}_{2}$. Blend compositions correspond to: Ti-64 (2), Ti+10\% TiC MMC (3), Ti+10\%TiB MMC (4), $\mathrm{Ti}+20 \% \mathrm{TiB} M M C(5)$. The shaded area on the chart denotes the dehydragination interval.

The difference in shrinkage behavior of layered plates with TiC and TiB can be explained by the different ways those two types of particles evolve within the structures. TiC has very high melting point $\left(3140^{\circ} \mathrm{C}\right)$ and doesn't undergo changes during the sintering. However, $\mathrm{TiB}$ is a result of in-situ reaction. Dilatometry data (Fig 3 ) show shrinkage, of $\mathrm{TiH}_{2}$ powder (9.5-10\%) with the temperature raise up to $1200^{\circ} \mathrm{C}$ (1). Shrinkage of TiC composite is at close value (3). For TiB composites ( 4 and 5) we observe slight elongation instead of shrinkage above $800{ }^{\circ} \mathrm{C}$ where in-situ reaction on Ti monoboride formations starts. The shrinkage of TiB composite is smaller at higher content of TiB particles. We observe two different morphologies of boride particles: needles and irregular resemble "sea urchin". Images show pores at the base of needles (Fig 4). There is an extensive porosity observed around particles with the "sea urchin" morphology. Due to the large difference in mutual diffusion mobility of boron and titanium it creates the Kirkendall's porosity effect. The increased porosity of TiB composites causes swelling and reduces shrinkage. 


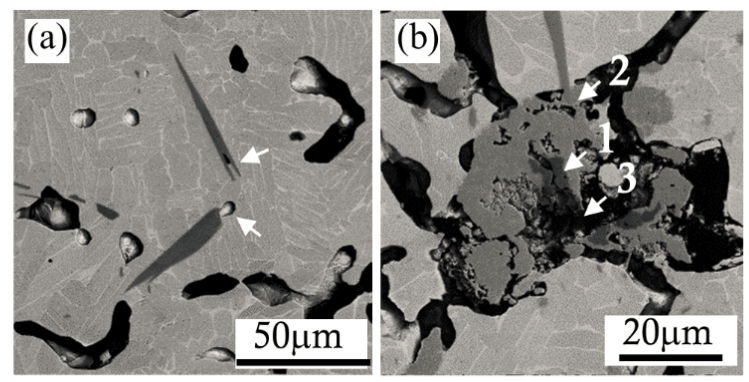

Fig. 4. SEM images within the TiB composite layer shown in the Fig. 1(a). Boride particles have two different morphologies: Needle (a) and "sea urchin" (b). The pores associated with needle formation are visible at the base of the needles, e.g. as arrowed in (a). Image (b) reveals internal structure of "sea urchin" particle showing the darker untransformed $\mathrm{TiB}_{2}$ at the center of the particle (1) surrounded by lighter $\mathrm{TiB}(2)$. The darkest contrast area in the middle of the particle is a pore (3). Image (b) demonstrate the extensive porosity surrounding the "sea urchin" particle.

To minimize shrinkage mismatch of individual layers during sintering of ML structures with TiB we tested several experimental parameters. The shrinkage of Ti-based compacts can be strongly affected by the hydrogen presence in powders [8]. We run the test on bi-layered bars to evaluate this effect. It was established that acceptable level of shrinkage mismatch can be reached when $\mathrm{TiH}_{2}$ powder is used for the composite layer and conventional CP-Ti powder for the alloy. However, it requires very high compaction pressure, $640 \mathrm{Mpa}$, to be used, which was not available in our laboratory set up for large-size plates pressing. The powder size is another well-recognized parameter to control the densification of sintered products. Our recent study on combined effect of the size and the type of the powder used shows that acceptable shrinkage mismatch can be achieved at $150 \mathrm{MPa}$ with proper selection of the hydrogenated titanium powder size used [7]. The ML plates with the size suitable for the ballistic test were fabricated using optimized parameters on the powder size and hydrogen content.

Some properties of studied materials are shown in the Table 1. Residual porosity of individual layers is strongly affected by the presence and type of reinforcement particles. Ti-64 alloy demonstrates the lowest porosity which depends on size of $\mathrm{TiH}_{2}$ powder used for fabrication of various $\mathrm{ML}$ structures. Porosity of TiC composite is some higher and the residual porosity of TiB composite is the highest. Vickers hardness and tensile properties are predetermined by the combined effect of residual porosity and the fraction of reinforcement particles. An increasing amount of strengthening particles causes the expected drop in elongation, while the anticipated increase in strength is not observed due to increased porosity and premature fracture. We observe some increase in Vickers hardness. However, the yield stress and ultimate tensile stress values of MMCs are substantially lower to those values of Ti-64 alloy sintered following optimized parameters (powder size, hydrogen content, compaction pressure) (item 7), shown here for comparison. 


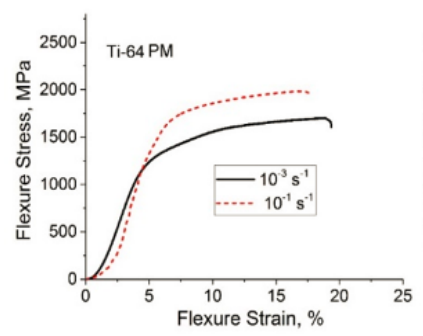

(a)

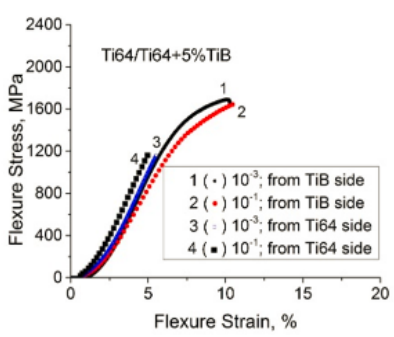

(d)

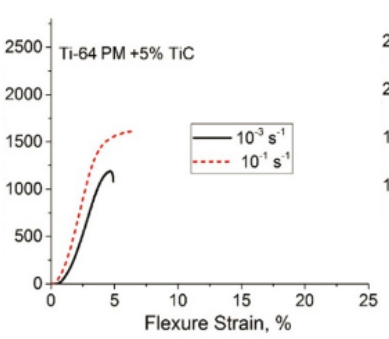

(b)

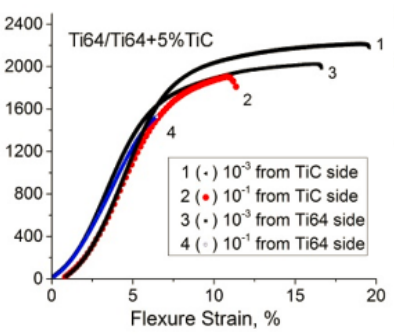

(e)

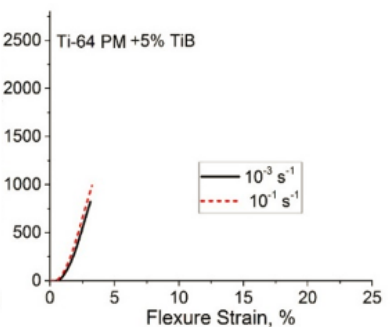

(c)

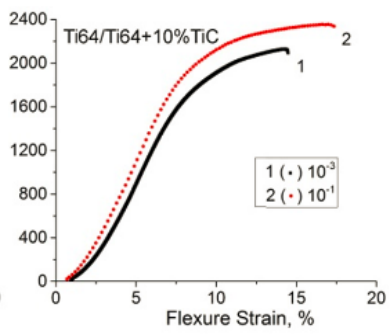

(f)

Fig. 5. Engineering stress-strain curves on 3-points flexure test of the uniform structures (a-c): alloy Ti-64 (a); Ti-64 + 5\% TiC (b); Ti-64 +5\% TiB (c). Bi-layered structures: (d) Ti-64 // Ti-64 $+5 \% \mathrm{TiB}$; (e) Ti-64 // Ti-64 + 5\% TiC; (f) Ti-64 // Ti-64 + 10\% TiC.

Results on 3-point flexure test (Fig 5) show substantial increase in both, the flexure stress and the flexure strain of bi-layered samples compared to a single-layer composite samples as well as the uniform Ti-64 alloy. For instance, bilayered sample with 5\% of TiC (Fig.5 (e) curve 2) compared to the uniform structure of 5\% TiC MMC in Fig.5(b) (red dotted line) demonstrates the increase of flexure stress on 25 and stain on $50 \%$. The change even more dramatic on bi-layered sample with $5 \% \mathrm{TiB}$ composite. For the same strain rate $\left(10^{-1} \mathrm{~s}^{-1}\right)$ we can see an increase of the flexure stress and flexure strain for bi-layered samples compared to uniform composite on about $63 \%$ and $300 \%$ correspondingly. (Fig. 5 (d) compared to Fig.5 (c)). Moreover, the highest flexure stress and strain values on a singlelayer samples demonstrates Ti-64 alloy (Fig. 5 (a)). When it compared to the best bi-layered samples values at the same strain rate $\left(10^{-1} \mathrm{~s}^{-1}\right)$ with $10 \%$ TiC $(\sim 2350 \mathrm{MPa})$ (Fig. 5(f)) we see close to $20 \%$ improvement of the flexure stress with no loss in the strain value. These results clearly show advantage of bi-layered vs. single layer structures. The measured deformation energy (integrated area under the stress-strain curves) at different strain rates allowed to directly relate results of 3-point flexural test with results of ballistic impact test on material [10]. It was noted [i.q.] that at higher strain rate the deformation energy of bi-layered structure with $10 \% \mathrm{TiC}$ is higher than that of Ti64 alloy. Also, stress/strain characteristics and deformation energy is better-quality for ML structures with TiC composites than with TiB ones (Fig. 5). This result implies bi-layered structure of Ti-64//Ti-64+10\% TiC can have better anti-ballistic properties compared to the uniform single-layer Ti-64 and ML structures with TiB prepared with used powder approach since it capable absorbing higher energy during deformation. 
Results on antiballistic test corroborates with the data on 3-point flexure test (Fig 6). We can see substantially better anti-ballistic performance of the layered structures compared to the cast Ti-64 alloy. Plates of about the same thickness, $25-27 \mathrm{~mm}$, were tested in similar conditions. Bullets of 7.62 caliber shoot the cast plate all the way through and stuck inside of the layered structures without showing damage on the back side of the tested plate. The cast sample on this slide was annealed at $1100{ }^{\circ} \mathrm{C}$ for $1 \mathrm{~h}$ and cooled with the furnace. That produced a lamellar microstructure [11].
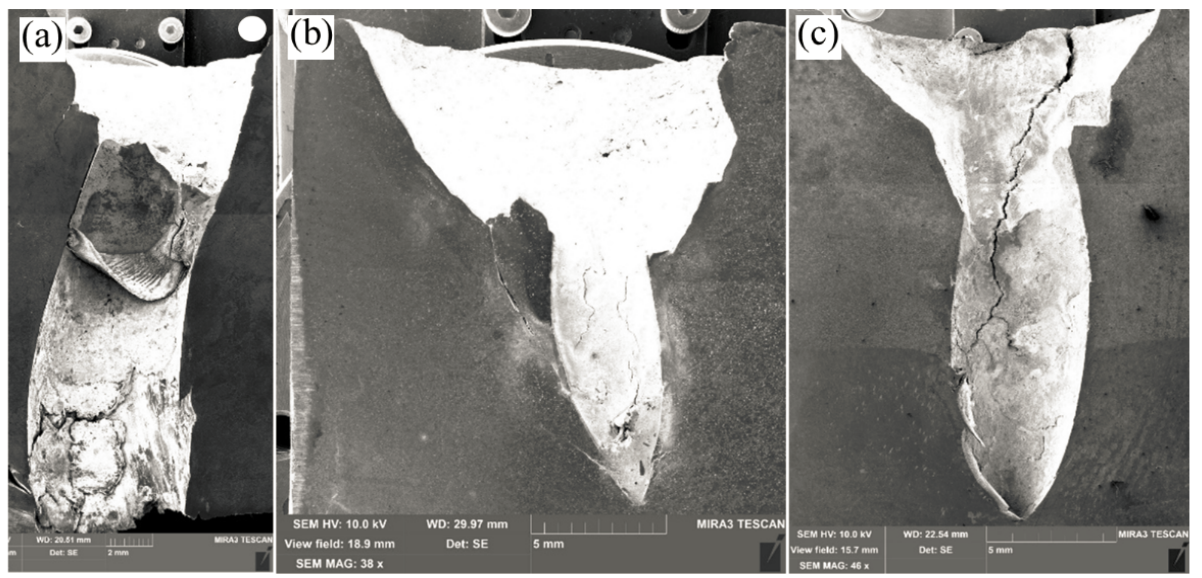

Fig. 6. Ballistic test results: (a) cast Ti-64 alloy; (b) bi-layered plate of Ti-64 // Ti-64 + 10\% TiB; (c) three-layered plate of Ti-64 // Ti-64 + 10\% TiC // Ti-64 + 5\% TiC. All plates have about the same thickness 25-27 mm. Test conditions: B-32 bullet (caliber 7.62X54 mm, mass $10.4 \mathrm{~g}$ ) kinetic energy 3500-3800 J.

We believe that residual porosity in as-sintered layered structures can have harmful effect on performance of materials (Table 1). The data show loss of Vickers hardness with increased porosity, even in the presence of highmoduli particles of $\mathrm{TiC}$ and $\mathrm{TiB}$ that supposed to contribute to the hardness gain. That is why we attempted hot plastic deformation of sintered ML structures for their more complete densification aiming in hardness improvement. Ideally such treatment should provide the porosity reduction without imposing uncontrollable grain growth and layers' delamination. It is well recognized that the hot deformation above $\alpha+\beta \otimes \beta$ transus temperature $\left(990{ }^{\circ} \mathrm{C}\right.$ for Ti-64) significantly softens the alloy and eases its deformation. However, it increases the risk of uncontrollable grain growth in part due to porosity reduction and gives higher affinity for the alloy's oxidation. For that reason, post-sintering hot plastic deformation of different structures was conducted at temperatures not far from the beta-transus of Ti-64 alloy. The applied temperature was ranged from $800-900^{\circ} \mathrm{C}$ when porosity of the samples was relatively low and up to $1100{ }^{\circ} \mathrm{C}$ for the samples with the highest porosity followed by annealing at $850^{\circ} \mathrm{C}$ for 2 hours. We found that the deformation of layered structures using hot rolling was unsuccessful due to significant disparity in metal flow and degree of accommodated plastic deformation in Ti-64 alloy and adjacent composite layers. As a result, significant distortion of the samples and delamination of the layers were observed [6]. We established the hot pressing of the plates were more successful. Such geometry of the load application in combination with the optimized temperature and deformation parameters provided shape preservation and 
integrity of the plates. ML samples with TiC particles were successfully hot pressed at $1100^{\circ} \mathrm{C}$ to a total deformation degree of $45 \%$, without breaking the integrity between the layers. At such conditions the deformation degree of Ti64 alloy layer in the ML structure reached $65 \%$ that resulted in reduction of total porosity of this layer to less than $1 \%$ and decrease in the pore size. The deformation behavior of the ML plates used MMCs with TiB was fairly different. The hot pressing was performed at the same temperature of $1100{ }^{\circ} \mathrm{C}$ and was stopped at $10 \%$ of the total deformation due to the cracks formation started within the MMC layers. At such degree of total deformation of the layered plate the layer of Ti-64 alloy was enable of $20 \%$ deformation and porosity reduction from 3.6 to $1-1.5 \%$. At such porosity level the grain growth was not critical leading to the average grain size of about $150 \mu \mathrm{m}$. MMC layers with TiB particles were barely deformed (no more than 5-10\%) and demonstrated minor decrease in porosity. Thermomechanical processing of the ML structures is still ongoing study, but our current results show the potentials on layered structures hardness increase after the optimized hot plastic deformation applied. We expect further improvement of antiballistic performance of $\mathrm{ML}$ structures especially with TiC composites. We also believe that an alternative approach on application of thermomechanical treatment can be used to improve the performance of the layered structures. In such approach optimized thermomechanical treatment could be used separately on each layer followed by layers bonding, i.g., by using diffusion bonding, HIP or friction welding etc.

\section{Conclusions}

Relatively big size layered structures of Ti-64 alloy and MMC on its base with TiC and TiB were successfully fabricated using BEPM die-pressing and sintering protocol. Sintered materials were characterized with uniform structure and composition within each layer and complete integration between the layers. During BEPM processing the shrinkage levels of the base alloy and MMC with TiC are similar, enabling the successful fabrication of multilayered structures without need for optimization of the sintering processing parameters for relatively large plates. The shrinkage level of MMC reinforced with TiB is significantly different from that of the alloy layer. Effective control of shrinkage mismatch between individual layers can be achieved through the careful selection of base powder size and the use of hydrogenated Ti powders.

Multi-layered as-sintered plates fabricated in the course of this study using BEPM were successfully tested for antiballistic application. Good antiballistic properties of ML plates were explained by experimentally measured increase of the flexure stress and flexure strain of the layered structures compared to uniform single layer structures.

We found that hot plastic deformation of layered structures has potentials on improvement of their mechanical properties and antiballistic performance. However, the deformation of the layered structures using hot rolling was unsuccessful due to disparity in the plastic flow of different layers. Hot pressing deformation at $1100{ }^{\circ} \mathrm{C}$ followed by the annealing was successfully applied to reduce the porosity of as-sintered layered structures with TiC MMCs and improve their hardness. The effect of hot pressing and annealing on the properties of the layered plates with TiB MMCs was less apparent.

Acknowledgment This study was supported by NATO Science for Peace and Security program (G5030) 


\section{References}

[1] Cimpoeru, S.J. The mechanical metallurgy of armour steels. Fishermans Bend, Victoria (Australia): Australian Department of Defence; Land Division, Defence Science \& Technology Group;(2016) Oct. Report No.: DST-Group-TR3305.

[2] El-Bitar, T., El-Meligy, M., El-Shenawy, E., Almosilhy, A., Dawood, N., Int J Mater and Metall Eng, (2017) 11(3), 214220.

[3] Montgomery, J.S., Wells, M.G.H., Roopchand, B., Ogilvy, J.W., JOM, (1997) 49(5), 45-47.

[4] Pettersson, A., Magnusson, P., Lundberg, P., Nygren, M., Int J Impact Eng, (2005) 32, 387-399.

[5] Lutjering, G., Williams, J.C., 2007. Titanium. Springer: Berlin, Heidelberg, NewYork.

[6] S.V. Prikhodko, P.E. Markovsky, D.G. Savvakin, O.O. Stasiuk, O.M. Ivasishin, Materials Science Forum, (2018) 941 1384-1390.

[7] O.M. Ivasishin, P.E. Markovsky, D.G. Savvakin, O.O. Stasiuk, M. Norouzi Rad, S.V. Prikhodko, Journal of Materials Processing Tech. (2019) 269 172-181.

[8] O.M. Ivasishin, V.S. Moxson, 2014. Low Cost Titanium Hydride Powder Metallurgy. In: Ma Qian and F.H. Froes (Eds.) Titanium Powder Metallurgy: Past, Present and Future, Elsevier.

[9] O.M. Ivasishin, D.G. Savvakin, V.S. Moxson, V.A. Duz, C. Lavender. Ti-2007 Science and Technology: Proceedings, 11th World Conf on Titanium (Kyoto, Japan), Eds. M.Niinomi, S.Akiyama et al., Sendai: Japan Institute of Metals, (2007) 1, 757-760.

[10] P.E. Markovsky, D.G. Savvakin, O.M. Ivasishin, V.I. Bondarchuk, S.V. Prikhodko, Journal of Materials Engineering and Performance (2019) (accepted)

[11] O.M. Ivasishin, S.V. Akhonin, D.G. Savvakin, V.A. Berezos, V.I. Bondarchuk, O.O. Stasyuk, P.E. Markovsky, Progress in Physics of Metalls, (2018) 3(19) 309-334. 